ethnic group. Results: $25 \%$ reported healthy weight (BMI $=18.5-24.9$ ); $45 \%$ overweight (BMI $=25.0-29.9)$; and $19 \%$ and $7 \%$ obesity categories I or II+ (BMI=30.0-34.9, 35+). In multivariable analyses controlling for sociodemographics and health care characteristics, we found significant differences in health behaviors and use of preventive services by categories of BMI. Obese men reported lower levels of alcohol consumption and were less likely to be a current smoker. On the other hand, overweight and obese men were less likely to consume a diet with $<30 \%$ of calories from fat [OR (CI), $0.71(0.68-0.74) ; 0.49(0.46-0.51) ; 0.38(0.35-0.41)]$. A similar trend was seen for physical activity. Overweight and obese men were less likely to participate in moderate activity [OR (CI), $0.81(0.78-0.84) ; 0.51(0.48-0.53)$; $0.31(0.29-0.33)]$ or vigorous activity [OR (CI), 0.73 (0.70-0.76); $0.47(0.44-0.50) ; 0.29(0.26-0.32)]$. An inverse trend was found for sedentary behavior. Men with a BMI of 35+ were twice as likely to report sedentary behavior for $6.5+$ hours/day [OR (CI), 2.04 (1.90-2.18)]. For screening exams, overweight and obese men were more likely to report having had their glucose, cholesterol, and triglycerides tested, and among men with diabetes, their HgAlc. In contrast, obese men were less likely to report a PSA test [OR (CI), $0.84(0.79-0.89) ; 0.74(0.68-0.80)]$ or, among men at average risk for colorectal cancer, a screening sigmoidoscopy [OR (CI), 0.84 (0.79-0.90); $0.73(0.67-0.81)]$. Conclusions: Despite having more primary care visits, obese men may be at greater risk due to lower utilization of common screening exams. Results suggest targeted efforts are needed to increase cancer screening and physical activity, and lower consumption of dietary fat among patients with elevated BMIs.

\section{Abstract C-A4-04}

One-Year Weight Change and Glycemic Control After Diagnosis of Diabetes Mellitus Type 2

Adrianne C. Feldstein, MD, MS, Center for Health Research, Kaiser Permanente Northwest; Gregory A. Nichols, PhD, Center for Health Research, Kaiser Permanente Northwest; David S. Smith, RPh, MHA, $\mathrm{PhD}$, Center for Health Research, Kaiser Permanente Northwest; A. Gabriela Rosales, MS, Center for Health Research, Kaiser Permanente Northwest; Nancy Perrin, PhD, Center for Health Research, Kaiser Permanente Northwest

Background/Aims: Limited real-world data describe weight change, associated factors, and glycemic control after diagnosis of diabetes. This study evaluated weight trajectories, glycemic control, and associated factors in the first year after diagnosis of diabetes mellitus type 2 in otherwise healthy patients. Methods: Participants were patients aged 21-75 with diabetes mellitus type 2 diagnosed between January 1, 1997 and December 31,2004 , identified from electronic medical records. Eligible patients for this retrospective cohort study met weight measurement criteria and did not have a condition associated with unintentional weight change $(n=4135)$. We estimated 12-month patient weight trajectories using growth curve analyses, grouped similar trajectories using cluster analysis, and compared characteristics among groups. Results: The four weight trajectory groups were 'higher stable weight' ( $\mathrm{n}=757 ; 18.3 \%)$, 'lower stable weight' $(\mathrm{n}=2236$; $54.1 \%)$, 'weight gain' $(\mathrm{n}=664 ; 16.0 \%)$, and 'weight loss' $(\mathrm{n}=478 ; 11.6 \%)$. Most patients (65.9\%) had some weight loss by 8 months and then regained. After adjustments, weight losers were more likely than gainers to be older, be female, take fewer medications, have had nutritionist visits, and have lower mean glycosylated hemoglobin (HbAlc) levels. Weight losers were less likely to be in a race group at higher risk for obesity, have depression or dyslipidemia, or have taken more than 30 days of a sulfonylurea alone or in combination with metformin. Conclusions: Most diabetes patients lost some weight after diagnosis. Weight loss trajectories were strongly associated with better glycemic control when compared to weight gain. Patients with certain characteristics may need more support for weight loss, and most need support for weight loss maintenance.
Abstract C-A4-05

US Immigration is Associated With Obesity Among Asian-Pacific Hotel Workers in Hawaii

Rachel Novotny, PhD RD, Kaiser Permanente Center for Health Research Hawaii and Univ. Hawaii; Andrew E. Williams, PhD, Kaiser Permanente Center for Health Research Hawaii; Aleli C. Vinoya, BS, Kaiser Permanente Center for Health Research Hawaii; Thomas M. Vogt, MD MPH, Kaiser Permanente Center for Health Research Hawaii

Background/Aims: The Work, Weight and Wellness (3W) program is a randomized trial funded by the National Institutes of Health, National Heart, Lung and Blood Institute. The program aims to decrease obesity among hotel workers in Hawaii. In this study we examined patterns of obesity in relation to migration status and ethnicity at baseline. Methods: Four thousand five hundred thirty hotel workers in 31 hotels were measured for weight, height and waist circumference at baseline, prior to intervention. Ethnicity and place of birth were self-reported. Data analysis was done using SAS. Results: Forty-five percent of hotel workers were born outside of the U.S. Of hotel workers born in the U.S., $35 \%$ were born in Hawaii, while $9 \%$ were born on the U.S. mainland. Among hotel workers born outside of the U.S., $38 \%$ were born in the Philippines, 3\% in Japan, 3\% in Southeast Asia, 1\% in other Pacific islands, and $12 \%$ in other locations. Self-reported ethnicity was $44 \%$ Filipino, 32\% other Asian, 13\% Pacific Islander, 9\% White and 3\% other ethnicity. The body mass index (BMI) of immigrants was $2.2 \mathrm{~kg} / \mathrm{m}^{2}$ higher than that of non-immigrants, on average, and waist circumference was $5.1 \mathrm{~cm}$ higher, on average. The BMI of males was greater than that of females and the interaction of sex and immigration on BMI was significant. The BMI of Pacific Islanders and Filipinos was greater than that of Whites, while BMI of Asians was lower than that of Whites; however, Filipino BMI was no longer greater than White BMI after adjusting for migration status and sex. Being immigrant, male and Pacific Islander was associated with greater BMI, while being Asian was associated with lower BMI. Conclusions: Birth outside of the U.S., male sex, Pacific Islander ethnicity and Filipino ethnicity were associated with obesity. Future study will examine the differential effects of the $3 \mathrm{~W}$ intervention program on obesity, and if this was modified by migration status, sex and ethnicity.

\section{Cancer}

\section{Abstract C-B1-02 \\ Spiritual Quality of Life of Long-Term Colorectal Cancer Survivors With Permanent Intestinal Stomas}

Mark C. Hornbrook, PhD, Center for Health Research, Kaiser Permanente Northwest; Carol M. Baldwin, PhD, RN, Arizona State University; Christopher Wendel, MS, Southern Arizona VA Health Care System; Marcia Grant, PhD, $\mathrm{RN}$, City of Hope National Medical Center; Michelle Ramirez, PhD, University of the Sciences; Carmit McMullen, $\mathrm{PhD}$, Kaiser Permanente Northwest; Lisa Herrinton, PhD, Kaiser Permanente Northern California; Jane Mohler, PhD, University of Arizona; Robert S. Krouse, MD, Southern Arizona VA Health Care System

Background: Religious and spiritual beliefs can influence adjustment to medical treatments and outcomes. This mixed methods cross-sectional study examined spiritual quality of life (QOL) of long-term colorectal cancer survivors with permanent intestinal ostomies. Methods: Men $(n=88)$ and women $(n=55)$ with ostomies whose total scores fell in the upper $(n=71)$ or lower ( $\mathrm{n}=72$ ) quartiles of the validated City of Hope Quality-of-Life ostomy-specific (COHQOL-O) survey provided quantitative and qualitative data regarding spiritual dimensions of QOL. Analyses included chi-square and analysis of variance \pm standard deviations with significances set at $P<0.05$. Content analysis was used to explicate meanings for spirituality items in survey narratives and focus groups. Results: The high COHQOL-O group was significantly more likely to be older $(74 \pm 9$ vs. $70 \pm 1.3, P<0.01)$ and married or partnered ( $72 \%$ vs. $55 \%, P<0.03)$. Upper quartile subjects had more favorable scores for the spiritual items Sense of Inner Peace, Hopeful, Reason to be Alive, and support from spiritual (e.g., prayer) and religious (e.g., church) activities compared to the lower quartile (all $P<0.0001$ ). The two groups did not differ for the item Positive Changes in life related to having an ostomy. Qualitative comments made by participants reflected the meaning of their high or low spiritual QOL scores. Conclusions: Spiritual 